\title{
Technological challenges of teaching mathematics in a blended learning environment
}

\section{Petr Sojka*}

Faculty of Informatics, Masaryk University, Brno 60200, Czech Republic E-mail: sojka@fi.muni.cz

*Corresponding author

\section{Roman Plch}

Faculty of Science, Masaryk University, Brno 61137, Czech Republic

E-mail: plch@math.muni.cz

\begin{abstract}
This paper describes the following technological aspects of blended learning of mathematics: effective preparation of electronic teaching materials suited for different students' needs (PDF, HTML and XML + MathML parallel generation), videotaping of mathematics lectures, automated (self)testing of subjects taught using the computer algebra system Maple and preparation of interactive teaching materials with the MapleNet technology. Authors describe results achieved and the experience gained during preparation and implementation of these challenges in a Calculus course taught at the Faculty of Science, Masaryk University in Brno, in autumn 2006.
\end{abstract}

Keywords: blended learning; calculus; format conversion; Maple; MapleNet; maplets; MathML; PDF; publishing technologies; teaching mathematics; technological challenges; TEX; tex4ht; video.

Reference to this paper should be made as follows: Sojka, P. and Plch, R. (2008) 'Technological challenges of teaching mathematics in a blended learning environment', Int. J. Continuing Engineering Education and Life-Long Learning, Vol. 18, Nos. 5/6, pp.657-665.

Biographical notes: Petr Sojka received his $\mathrm{PhD}$ in Computational Linguistics at the Masaryk University, Brno, Czech Republic. Currently, he is an Assistant Professor at the Faculty of Informatics, Masaryk University in Brno. His main research interests include electronic publishing, textual information systems and blended learning support. He has published over 70 papers.

Roman Plch received his $\mathrm{PhD}$ in Mathematics at the Masaryk University, Brno, Czech Republic. Currently, he is an Assistant Professor in the Faculty of Science, Masaryk University in Brno. His main research interests include computer algebra systems, technology in teaching mathematics and blended learning support.

Copyright (C) 2008 Inderscience Enterprises Ltd. 


\section{Motivation}

Teaching of mathematics is specific as are the technological challenges of our digital age for its support. Teaching of mathematics has its traditions, settled over centuries and glorified by traditional professors, in stark contrast to the possibilities of Information Communication Technologies (ICT) change every year.

We have prepared several electronic materials and tried several new approaches to support students of mathematics at Masaryk University in Brno, who are enrolled in a Calculus course.

\section{Objectives and their realisation}

The Information System of Masaryk University (http:/is.muni.cz, IS MU) is currently being enhanced to support blended learning methods which includes an extensive usage of ICT. It allows authenticated access to structured study materials, there is support for students' (self-)testing and examination, and new functions are continuously being added with the aim of it becoming fully fledged Learning Management System (LMS). As several specific needs for supporting the teaching of mathematics were missing, we have investigated the possibilities of their realisation in the pilot project of support of the course M3501 Calculus III (Metric spaces and Calculus of several variables) in the autumn semester 2006.

\subsection{Study materials in different formats}

Every student is different. 'Put yourself in the reader's place,' is the oft repeated incentive. Students study by reading the course materials on their notebook or computer screens, doing exercises with pen and paper or using a Computer Algebra System (CAS) on the computer, testing themselves by solving special tests in IS MU or even hearing and watching the lectures repeatedly.

Mathematics textbook authors prefer writing their text and exercises once only (usually in TEX), rather than changing their setup every semester. On the other hand, there are the new possibilities: of high resolution computer screens, internet and PC availability almost everywhere by almost every student. New formats brings new possibilities: PDF allows high fidelity of textbook delivery, MathML (or OMDoc) soon to be accepted as the structured text format for exchanging and delivery of mathematics electronic documents. On the other hand, TEX notation remains preferred by authors for editing and authoring.

For Calculus III course there were two textbook materials prepared in LaTeX, i.e. Došlá and Došlý (2000) and Došlá, Plch and Sojka (1999). We have evaluated that at least four formats are of interest to the students:

1 PDF suitable for printing: electronic copy of the printed textbooks (no colours).

2 PDF suitable and designed for PC screen reading: PC screen has different aspect ratio, resolution, allows usage of colours and easy searching.

3 HTML for reading in the 'old-fashioned' web browsers, with math formulas as pictures.

4 XHTML/MathML for reading in 'new generation' web browsers, allowing 'cut\&paste' functionality with CAS systems. 
We have managed to automate the generation of all four formats of the two textbooks (more than 300 pages) from single LaTeX sources. We have used pdfTEX (http://en.wikipedia.org/wiki/PdfTeX) and tex4ht (http://www.cse.ohio-state.edu/ gurari/ TeX4ht/) programs.

We have added conditionals in the single LaTeX source file for every book to switch settings for these four versions, and prepared Makefile to automate the processes as much as possible.

PDF suitable for printing. Printing without colours is sufficient for both textbooks, however pictures need to be adjusted as high-resolution bitmaps or vectors only. Several versions were generated for different paper formats (A4 with wide margin for comments and B5). Navigation and references in the text were implemented by standard LaTeX citation macros, see Figure 1.

Figure 1 Design suitable for printing (PDF)

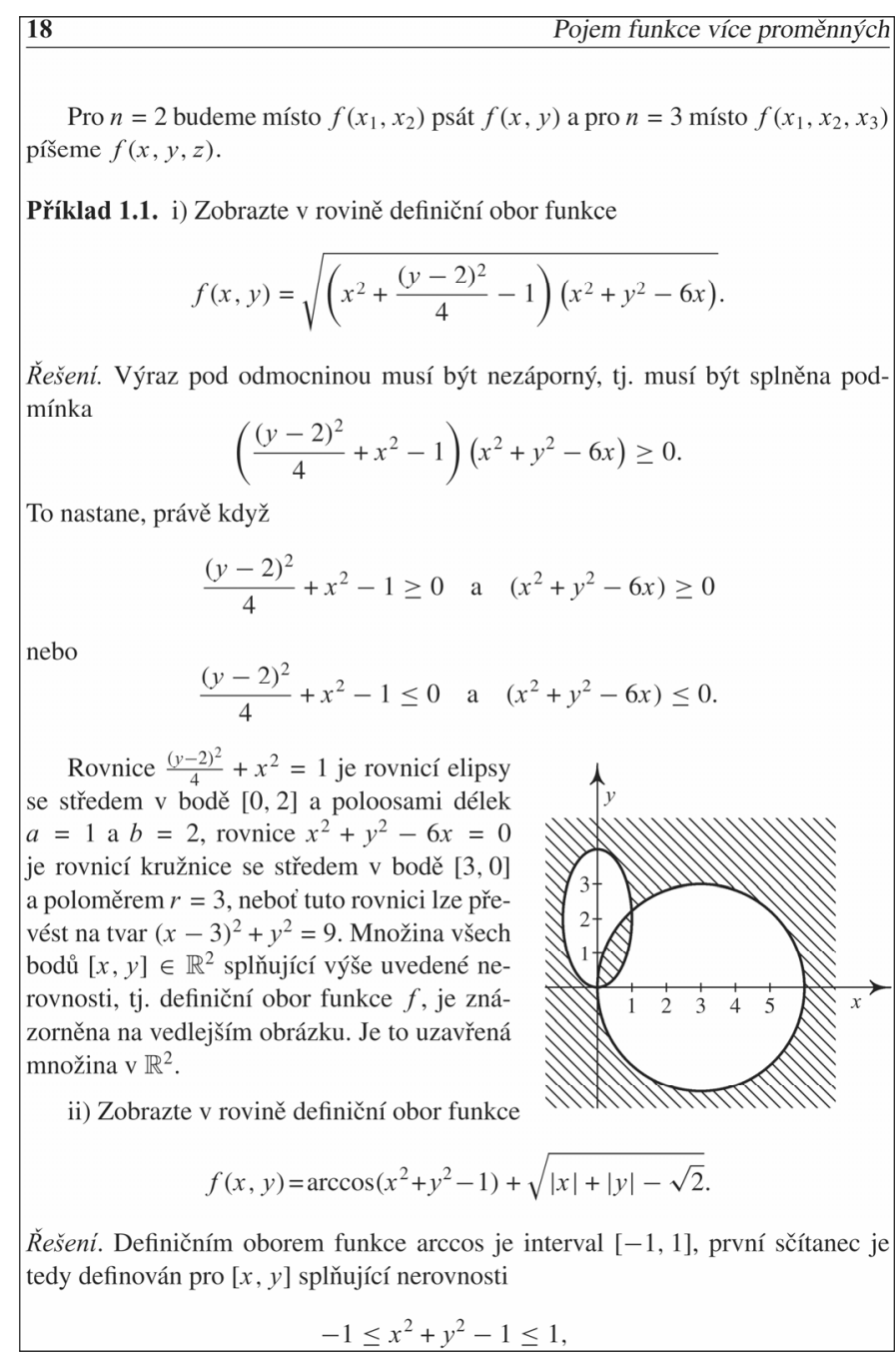


PDF designed for screen reading. A special version for reading on standard computer screen (width:height ratio 4:3, cca 100 ppi, colour) has been designed - see Figure 2. Colour is used extensively for the reader's navigation in the hypertext. Animations have been added too, based on the possibilities of embedded Java-Script in the PDF (Sojka, 2003).

Figure 2 Design for computer screen (PDF) (see online version for colours)

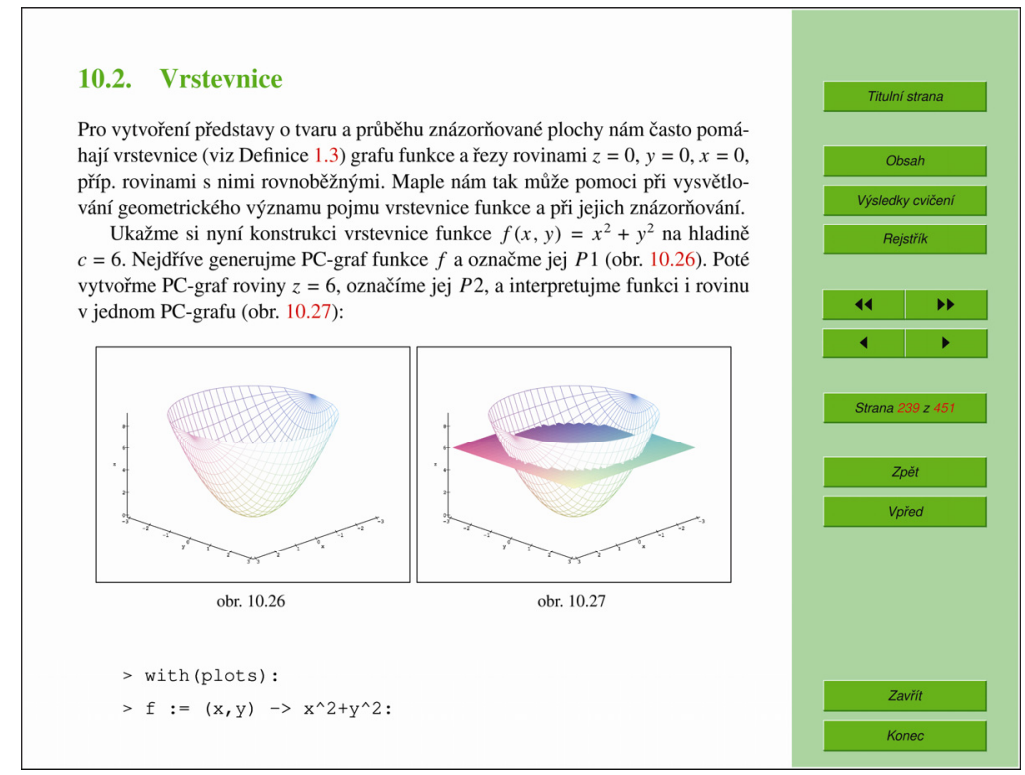

\subsubsection{Conservative HTML}

Having documents published as web pages in HTML 4 which is one of the safest and most foolproof options. We have tested several possible options for automatically generating HTML versions of the books from LaTeX sources: LaTeX2html, TEX4ht (http://www.cse.ohio-state.edu/ gurari/TeX4ht/), home-grown converter in Python, tex2page (http://www.ccs.neu.edu/home/dorai/tex2page/), hyperlatex, Hevea and others. We ultimately chose TEX4ht for its stability and power, although it is a rather complex system and has to be enriched for new authors' markup. For mathematics not expressible in HTML, small transparent images in PNG format are generated, see Figure 3.

\subsubsection{Progressive MathML}

MathML is a W3C proposal for math in XML on the internet, already supported by several browsers and almost all computer algebra systems. There are several tools converting TEX to present MathML (Tralics (http://www-sop.inria.fr/apics/tralics/), LaTeXML (http://dlmf.nist.gov/LaTeXML/), Omega and others). We have stayed with TEX4ht, as it can be configured for MathML generation as well. This version is especially useful for blind people, as math covered by MathML does not use any picture and is the text rendering scales well and quickly in web browsers. It fulfills Web Accessibility Initiative, http://www.w3.org/WAI (WAI) suggestions, see Figure 4. 
Figure 3 Design for web (HTML) (see online version for colours)

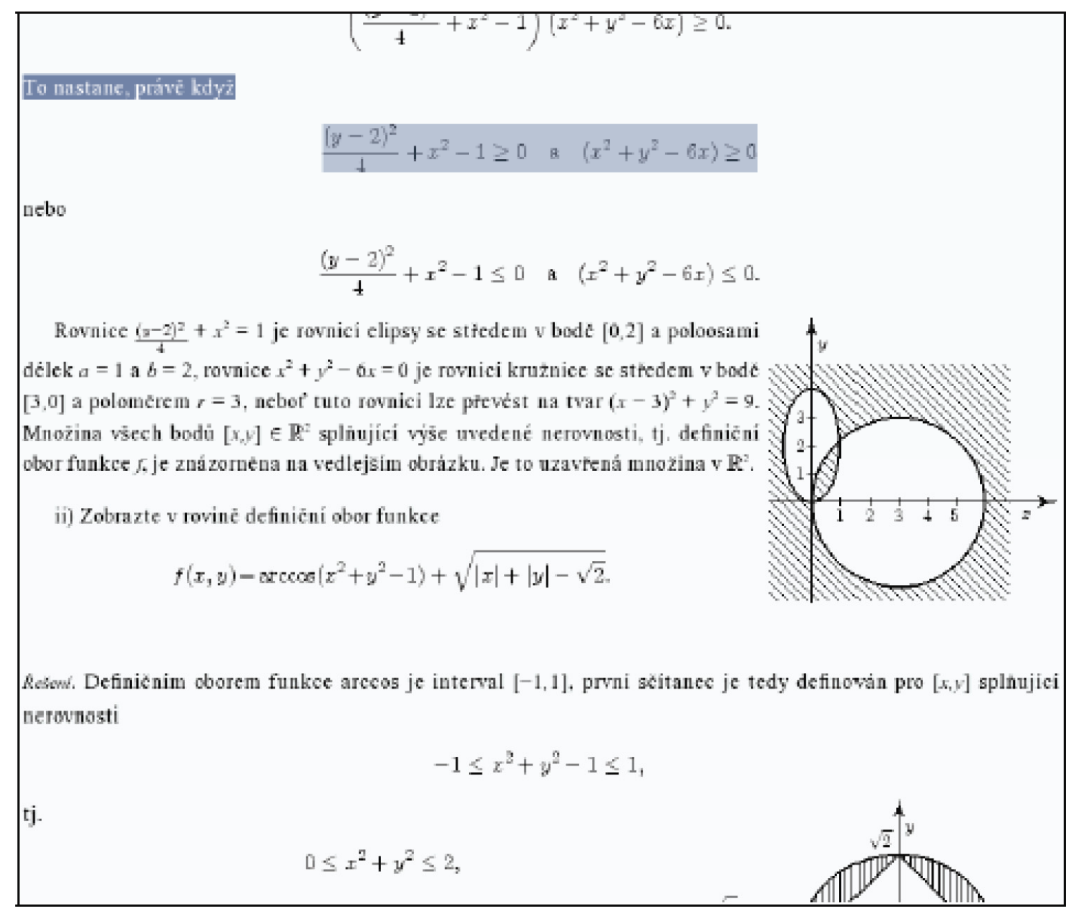

Figure 4 Design for web (MathML) (see online version for colours)

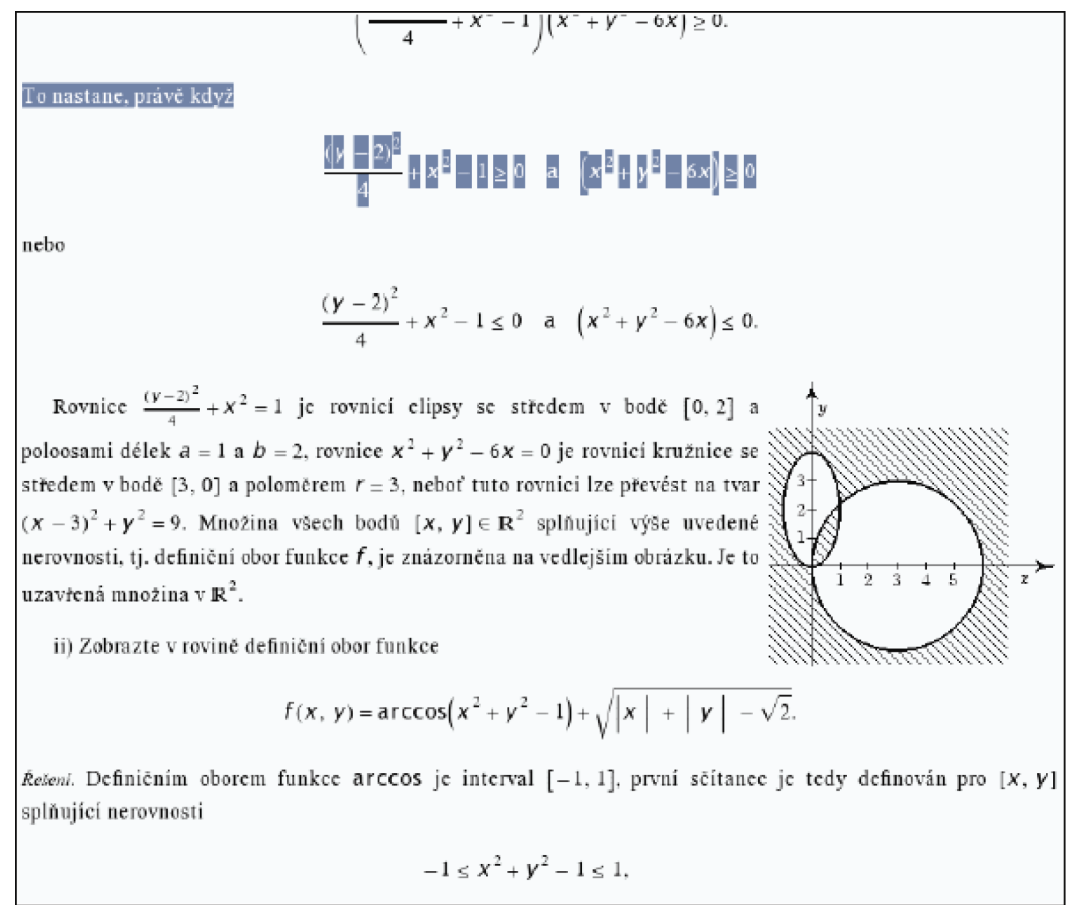


All conversions were done by free software and tools, available as part of the TeXlive project (http://www.tug.org/texlive). The support of MathML has not yet been fully implemented in all web browsers, but we expect that the situation will improve as the benefits are easily seen and widely accepted. We envisage that TEX notation will remain preferred in math authors community for authorship. This trend is supported by a plethora of tools supporting TEX math notation on the web (ASCIIMath http://www1.chapman. edu/ jipsen/asciimath.xml, JSMath http://www.math.union.edu/ $\sim$ dpve/jsMath/, IBM texplorer) or publishing systems that allow TEX notation for input (MathType plugin for Word, 3B2, etc.). For details see Sojka and Růžička (2007).

\subsection{Videotaping lectures}

Mathematics professors are used to deliver their lectures 'the good old way' using the blackboard table and chalk. This has several advantages: introducing new definitions and proof deductions cannot be increased to an incomprehensible speed of reading Powerpoint bulleted items Tufte (2003).

There are students who benefit from hearing the teacher, and those who have to see the teacher talking or writing on the board. We have videotaped all the lectures using the two 3CCD miniDV cameras (Canon DM-XM2). One view was from static camera and the second was by the cameraman taking the details of the teacher and the blackboard. The teacher uses a wireless micro-port microphone to grab high quality voice, but in general, very few constraints are imposed on the teacher. Every videotaped lecture is available in the AVI format to students in the IS within a few days. A short example of video has been put on the web so that reader may have closer look at current videotaping possibilities http://www.fi.muni.cz/usr/sojka/videos/M3501examples.avi.

Last but not least, we have experimented with Flash technology, where PDF and downsampled videos were loaded in separate windows. We made both materials mutually crosslinked - seeing a new page in PDF searches for an appropriate sequence in the video window and vice versa.

\subsection{Automated testing and evaluation by Maple}

The greatest obstacle to automated exercising in LMS IS (and to the best of our knowledge in other systems, too) is the automated evaluation of results of mathematical exercise and subsequent checking of the results. We have designed and implemented a new type of testing exercises using the computer algebra system Maple. It evaluates the math the student types into the browser form.

There is a standard function testeq in Maple for testing of equivalence, but it works only for simple algebraic expressions. For testing other mathematics objects we wrote a new Maple code, which is able to test algebraic expressions, lists, sets, matrices, vectors, etc. for equivalence. The corresponding Maple worksheet is available at http://www.math.muni.cz/ xsrot/mws/newtesteq.mws (Šrot, 2007). The benefit is, that it should be able to test also nested objects.

So that students were not tempted to cheat by using the powerful Maple functions (ad diff for derivation tasks), a system of allowed and forbidden function names (white-list and black-list) was introduced. The functions listed in the black-list are not allowed for an exercise solution. In the case where a teacher does not explicitly lists the function names allowed, a white-list is generated from the canonical solution. 
Figure 5 shows an Example test with results. The first question is solved by means of Maple function diff, which is not allowed in this case. The second question has a wrong answer. The third question is solved succesfully although the equation is written in a form different from the given result.

Figure 5 Example test with results (see online version for colours)

\section{Testing the new type of question (for testing mathematics)}

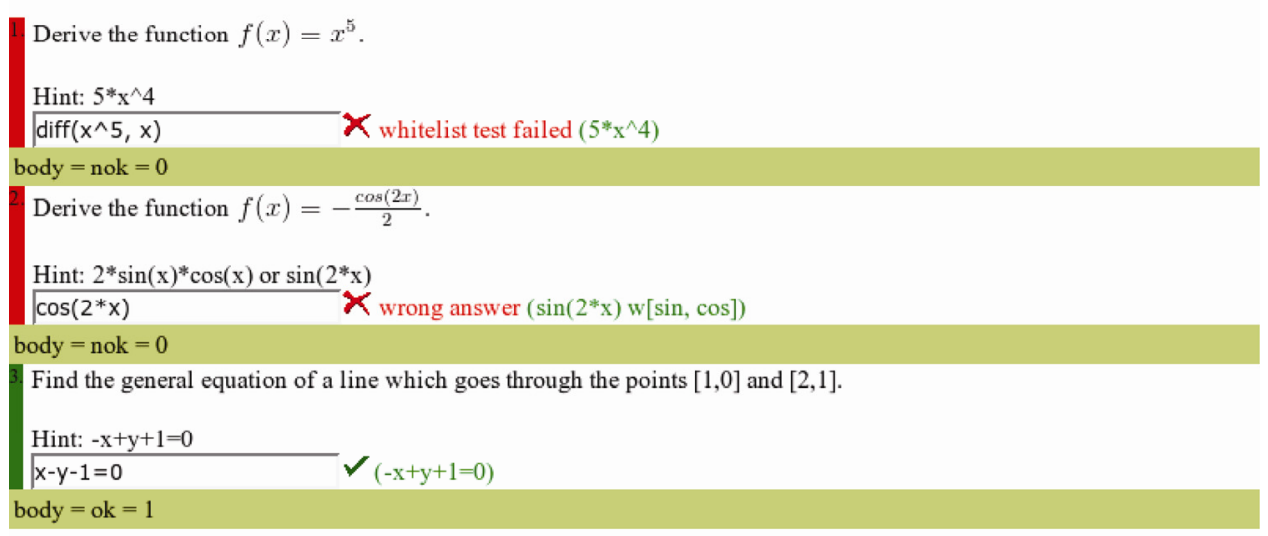

\section{End of the test}

Total score: 1 (total number of questions: 3 )

\subsection{Interactive study with MapleNet}

MapleNet (http://www.maplesoft.com/products/maplenet/) is a technology allowing on-the-fly Maple computations in web applications. With MapleNet, one can create interactive teaching materials with Java applets, Maplets or Maple worksheets. To read these materials, the user needs a web browser with Java support enabled and a plug-in client for connection to the MapleNet server. Students do not have to have Maple installed on their computer, but may use the power of symbolic computation on their desk: an important new portable tool for blended learning. Special interactive graphic applications called Maplets, written in the Maple programming language bring exciting new possibilities. Maplets are platform independent, thus we can use them on a computer with Windows operating system as well as on a Linux/Unix machine.

These interactive graphical applications were prepared for support of Calculus III course (functions of several variables, limits, partial and directional derivatives, differential, Taylor polynomilas, maximum and minimum values, Lagrange multipliers and implicit functions). In most cases, the user can display not only the final answer or the graphical output, but also all solution steps. Maplets are posted in LMS IS MU using the interactive structured curriculum tool. Their overview can be viewed on http://melian.ics.muni.cz:8080/maplenet/glozar/, Figure 6 shows partial derivatives maplet. The purpose of this Maplet is to help the user to compute and 
visualise partial and directional derivatives to a surface in three dimensions. The Maplet is directly accessible from the adress http://melian.ics.muni.cz:8080/maplenet/glozar/ ParcDerE:14444/. For details see Glozar (2007).

Figure 6 Partial Derivatives Maplet (see online version for colours)

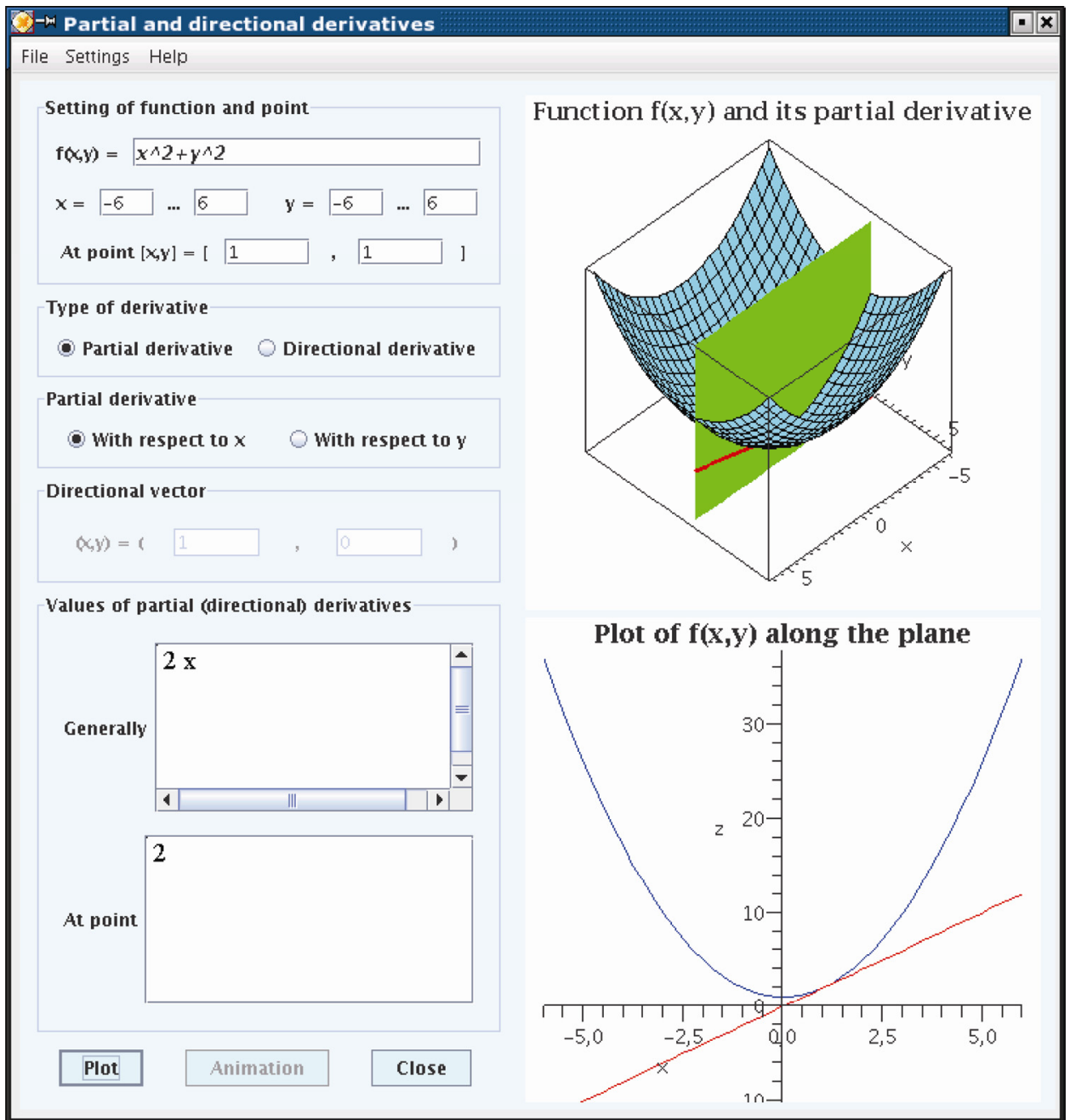

\section{Conclusion and further work}

Evaluation of the new generation of support for the Calculus course shows that new aspects of the of course are used by the students differently. New possibilities allow students to choose studying materials according to their preferences, skills and studying methods. Maplets are powerful visualisation tools that help with the understanding of covered topics. 
Stepping in the new technology e-learning possibilities is an adventurous exercise with many 'no way' experiences. However, once set up and the students start using them, we are certain that learning productivity increases considerably. Rather marginal changes in the teachers' uses of available technologies may have a drastic impact on the usefulness and accessibility of math teaching materials.

\section{Acknowledgements}

This work has been partially supported by grant 1ET208050401 of Academy of Sciences $\check{C} R$.

\section{References}

Došlá, Z. and Došlý, O. (2000) Metric Spaces: Theory and Examples (in Czech) (2nd ed.). Brno: Masaryk University in Brno, ISBN 80-210-1328-1.

Došlá, Z., Plch, R. and Sojka, P. (1999) 'Matematická analýza s programem Maple: 1. Diferenciální počet funkcí více proměnných (Mathematical Analysis with Program Maple: 1. Differential Calculus)'. Available at: http://www.math.muni.cz/ plch/mapm/.

Glozar, J. (2007) in P. Sojka and M. Kvizda (Eds), Maplety ve výuce matematiky (in Czech), p.21-26, ISBN 978-80-210-4296-4, Masaryk University. Available at: http://sco.muni.cz/2007/scoproc.html.

Sojka, P. (2003) 'Interactive teaching materials in PDF using JavaScript', Paper presented in the Proceedings of the 8th SIGCSE Annual Conference on Innovation and Technology in Computer Science Education, ITiCSE 2003, Association of Computing Machinery, Thessaloniki, p.275, ISBN 1-58113-672-2.

Sojka, P. and Kvizda, M. (Eds) (2007) SCO 2007 - Sharable Content Objects, Masaryk University, Brno, Czech Republic, May, ISBN 978-80-210-4296-4. Available at: http://sco.muni.cz/ 2007/scoproc.html.

Sojka, P. and Růžička, M. (2007) 'Single-source publishing in multiple formats for different output devices', Paper presented in the Proceedings of the EuroBachoT $E^{X}$ 2007, XVII. European $T_{E} X$ Conference, Polish $\mathrm{T}_{\mathrm{E}} \mathrm{X}$ Users Group GUST and Czechoslovak $\mathrm{T}_{\mathrm{E}} \mathrm{X}$ Users Group CSTUG, Bachotek, Brodnica, pp.125-131, ISBN 83-910954-3-6.

Šrot, K. (2007) in P. Sojka and M. Kvizda (Eds), Nový typ testové otázky v IS MU (in Czech), pp.21-26, ISBN 978-80-210-4296-4. Available at: http://sco.muni.cz/2007/scoproc.html.

Tufte, E.R. (2003) The Cognitive Style of PowerPoint. CT, USA: Graphics Press LLC, ISBN 0-9613921-54. 